\title{
Excess Low-Frequency Noise in Ultrathin Oxide n-MOSFETs Arising From Valence-Band Electron Tunneling
}

\author{
Jun-Wei Wu, Student Member, IEEE, Jian-Wen You, Huan-Chi Ma, Chih-Chang Cheng, Chang-Feng Hsu, \\ Chih-Sheng Chang, Gou-Wei Huang, and Tahui Wang, Senior Member, IEEE
}

\begin{abstract}
Low-frequency flicker noise in analog n-MOSFETs with $15-\AA$ gate oxide is investigated. A new noise generation mechanism resulting from valence-band electron tunneling is proposed. In strong inversion conditions, valence-band electron tunneling from $\mathrm{Si}$ substrate to polysilicon gate takes place and results in the splitting of electron and hole quasi-Fermi-levels in the channel. The excess low-frequency noise is attributed to electron and hole recombination at interface traps between the two quasi-Fermi-levels. Random telegraph signals due to the capture of channel electrons and holes is characterized in a small area device to support our model.
\end{abstract}

Index Terms-Low-frequency noise, random telegraph signal, ultrathin oxide MOSFET, valence-band tunneling.

\section{INTRODUCTION}

C MOS technology, which possesses the advantages of low cost, high integration, and low power, is finding more and more important applications in the area of mixed-mode and RF ICs. As compared with bipolar transistors, CMOS devices exhibit large noise, especially in the low-frequency domain where flicker noise is dominant [1]. Drain current flicker noise has become one of the key considerations in device geometrical scaling since it will affect the signal-to-noise ratio in operational amplifiers and in analog/digital and digital/analog converters. In addition, low-frequency flicker noise can be upconverted to undesired phase noise in RF circuits [2] and limits the channel spacing in communication systems. In order to reduce low-frequency noise, the physical origin of flicker noise in today's CMOS devices with gate oxide in direct tunneling regime should be further explored.

The origin of low-frequency flicker noise in MOSFETs with relatively thick gate oxides has been extensively studied. A unified noise model [3], [4] based on oxide charge tunnel trapping and detrapping has been adopted. The carrier number

Manuscript received December 2, 2004; revised May 18, 2005. This work was supported by the Taiwan Semiconductor Manufacturing Company (TSMC) and by the National Science Council, Taiwan, R.O.C., under Contract NSC92-2215E009-024.

J.-W. Wu, H.-C. Ma, C.-C. Cheng, C.-F. Hsu, and T. Wang are with the Department of Electronics Engineering, National Chiao-Tung University, Hsinchu 300, Taiwan, R.O.C.

J.-W. You is with the United Microelectronics Corporation, Hsinchu 300, Taiwan, R.O.C.

C.-S. Chang is with the Taiwan Semiconductor Manufacturing Company, Hsinchu 300, Taiwan, R.O.C.

G.-W. Huang is with the National Nano Device Laboratories, Hsinchu 300, Taiwan, R.O.C.

Digital Object Identifier 10.1109/TED.2005.854264 and mobility fluctuation resulting from trapped oxide charges is thought to be the source of flicker noise. In addition, some studies showed that the low-frequency noise may result from charge emission and capture at interface traps in weak inversion condition or in the very high frequency regime of noise power spectral density [5]. As gate oxide thickness is scaled into direct tunneling domain, oxide trap density should be much reduced. In addition, channel electrons would likely tunnel through an ultrathin gate oxide directly without being captured by oxide traps. However, the low-frequency noise in ultrathin oxide CMOS devices still exhibits a $1 / f$ spectrum and possesses a significant level [6], [7]. The traditional oxide charge tunnel trapping and detrapping concept seems no longer suitable to explain the noise behavior in ultrathin oxide MOSFETs. Recently, a study for ultrathin gate oxide fully depleted/partially depleted silicon-on-insulator (SOI) MOSFETs has shown that linear kink effect induced by valence-band electron tunneling would increase the low-frequency noise spectral density $S_{I}$ [8]. In this paper, we observe another source of low-frequency noise in ultrathin gate oxide bulk n-MOSFETs arising from valence-band electron tunneling [9], [10]. Detailed discussion on the physical mechanism of this excess noise will be given.

The time domain presentation of low-frequency noise is known as random telegraph signal (RTS) and has been studied in past decades [11]-[14]. Due to a single charge trapping and detrapping in a small area device, RTS exhibits two levels. The upper level corresponds to an empty trap, i.e., no electron occupation, and the duration of time is denoted by $\tau_{H}$. The lower level corresponds to an electron occupied state and is denoted by $\tau_{L}$. In many cases, $\tau_{H}$ corresponds to the time it takes to capture an electron, while electron release (emission) from traps governs $\tau_{L}$ [15].

In this work, the low-frequency noise in a $15-\AA$ gate oxide $\mathrm{n}$-MOSFET is investigated. The electron trapping and detrapping times $\left(\tau_{H}\right.$ and $\left.\tau_{L}\right)$ are characterized from RTS in a small area n-MOSFET. The power spectral density of normalized drain current noise $\left(S_{i d} / I_{d}^{2}\right)$ and gate referred voltage noise $\left(S_{\mathrm{vg}}\right)$ is also measured. In addition, the RTS time constants and noise power spectral density in $33 \AA$ oxide n-MOSFETs, where valence-band tunneling is insignificant, are characterized for comparison. The drain bias in RTS and noise measurement in this paper is $0.2 \mathrm{~V}$ to ensure a uniform charge distribution in the channel. Finally, a new noise source due to valence-band electron tunneling will be proposed to explain the observed noise behavior. 


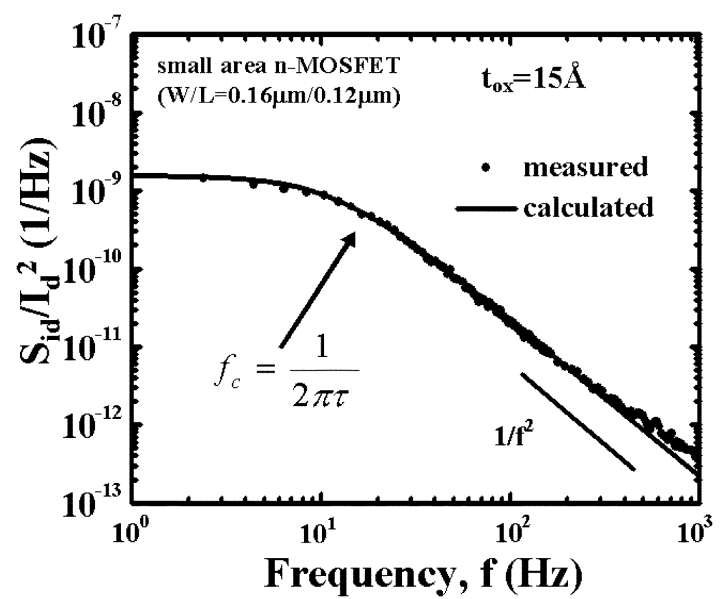

Fig. 1. Measured and calculated Lorentzian-like noise power spectral density in a small area n-MOSFET $\left(W / L=0.16 / 0.12 \mu \mathrm{m}, t_{\mathrm{ox}}=15 \AA\right)$. The noise is measured at strong inversion $\left(V_{d}=0.2 \mathrm{~V}, V_{g}=1.1 \mathrm{~V}\right)$. The cut-off frequency $\left(f_{c}\right)$ is also shown in the figure.

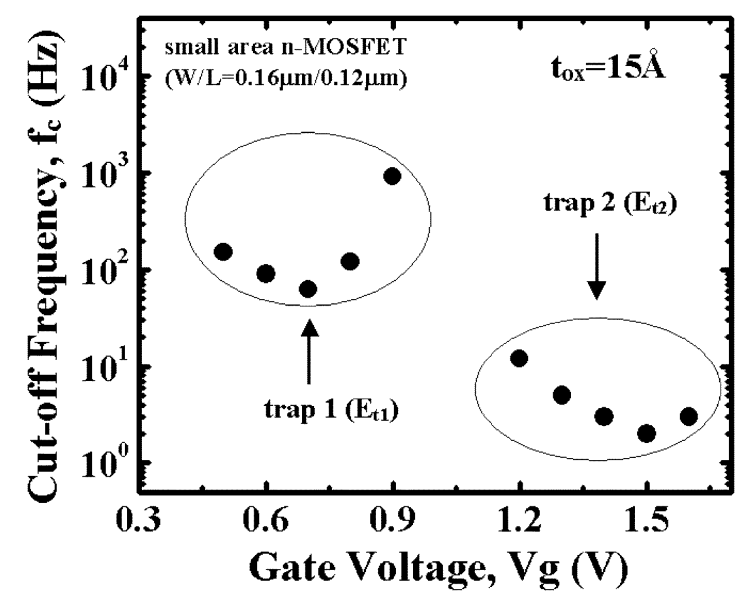

Fig. 2. Cut-off frequency versus gate voltage in a small area n-MOSFET $\left(W / L=0.16 / 0.12 \mu \mathrm{m}, t_{\mathrm{ox}}=15 \AA\right)$. A trap $\left(E_{t 1}\right)$ is observed in weak inversion $\left(V_{g} \sim 0.7 \mathrm{~V}\right)$ and another trap $\left(E_{t 2}\right)$ is in strong inversion $\left(V_{g}>1.0 \mathrm{~V}\right)$.

\section{RESULTS AND DISCUSSION}

The noise characteristic in a small area ultrathin oxide n-MOSFET with a single trap time constant is first analyzed. Fig. 1 shows the measured and calculated noise power spectral density in a $W / L=0.16 / 0.12 \mu \mathrm{m}$ n-MOSFET. The gate oxide thickness is $15-\AA$. The noise has Lorentzian-like spectral distribution [15], characterized by a constant power spectral density at low frequencies and a roll-off with $f^{-2}$ for high frequencies, .i.e.

$$
S_{i d} \propto \frac{\tau}{1+(2 \pi f \tau)^{2}} \text { and } \frac{1}{\tau}=\frac{1}{\tau_{H}}+\frac{1}{\tau_{L}} .
$$

The cut-off frequency $\left(f_{c}\right)$ corresponds to the $3-\mathrm{dB}$ point of the spectrum and is related to the reciprocal characteristic time $(\tau)$ of the underlying trap $\left(f_{c}=1 / 2 \pi \tau\right)$. The calculated result (solid line in Fig. 1) is based on $\tau_{H}$ and $\tau_{L}$ extracted from associated RTS (will be shown later) and is in good agreement with the measured power spectral density. Fig. 2 shows the gate voltage $\left(V_{g}\right)$ dependence of $f_{c}$. Obviously, there exist two groups of trap frequency (or two trap energy levels) with one $\left(E_{t 1}\right)$ observed

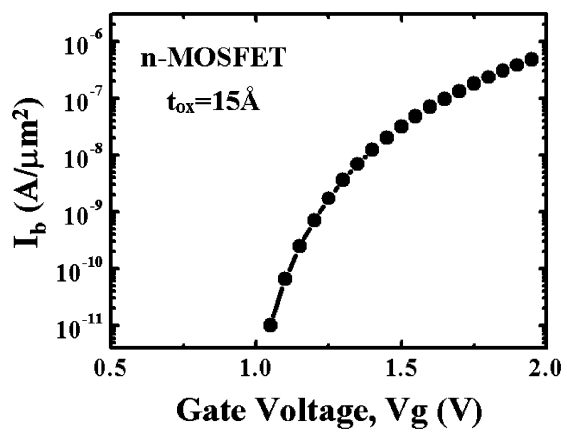

Fig. 3. Substrate current $\left(I_{b}\right)$ versus gate voltage in a $15-\AA$ oxide n-MOSFET. The $I_{b}$ in the $15-\AA$ oxide device drastically increases for $V_{g}>1.0 \mathrm{~V}$ (strong inversion regime), which indicates the occurrence of valence-band electron tunneling.

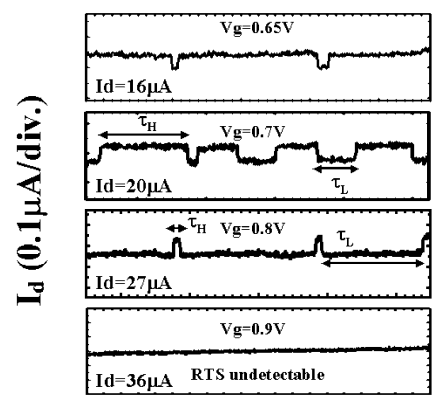

Time (0.5ms/div.)

(a)

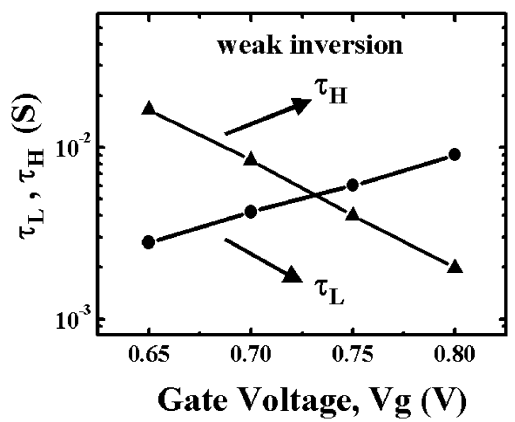

(b)

Fig. 4. (a) Typical RTS patterns at various gate voltages from $V_{g}=0.65$ to $0.9 \mathrm{~V}$ in a small area n-MOSFET $\left(W / L=0.16 / 0.12 \mu \mathrm{m}, t_{\mathrm{ox}}=15 \AA\right)$. RTS is undetectable at $V_{g}=0.9 \mathrm{~V}$. (b) Average $\tau_{L}$ and $\tau_{H}$ (extracted from RTS) versus gate voltage in weak inversion regime.

in weak inversion $\left(V_{g}<0.9 \mathrm{~V}\right)$ and the other $\left(E_{t 2}\right)$ in strong inversion $\left(V_{g}>1 \mathrm{~V}\right)$. Furthermore, significant substrate current arises in the $15-\hat{A}$ oxide device in strong inversion regime $\left(V_{g}>1 \mathrm{~V}\right)$ in Fig. 3 because valence-band electron tunneling from the Si substrate to the polysilicon gate occurs and generated holes flow to the substrate [16].

Fig. 4(a) shows typical RTS patterns in a small area $(W / L=$ $0.16 / 0.12 \mu \mathrm{m}) 15-\AA$ gate oxide n-MOSFET in weak inversion $\left(V_{g}<0.9 \mathrm{~V}\right)$. As can be seen, $\tau_{L}$ increases and $\tau_{H}$ decreases as $V_{g}$ increases from 0.65 to $0.9 \mathrm{~V}$. Noticeably, RTS vanishes at $V_{g}=0.9 \mathrm{~V}$ in our measurement period. Fig. 4(b) shows the $V_{g}$ dependence of average $\tau_{L}$ and $\tau_{H}$ (extracted from RTS) in weak inversion regime. The $\tau_{L}$ and $\tau_{H}$ in weak inversion correspond to the electron emission and capture times at the interface trap $E_{t 1}$, as illustrated in Fig. 5(a). As $V_{g}$ increases, $\tau_{H}$ 


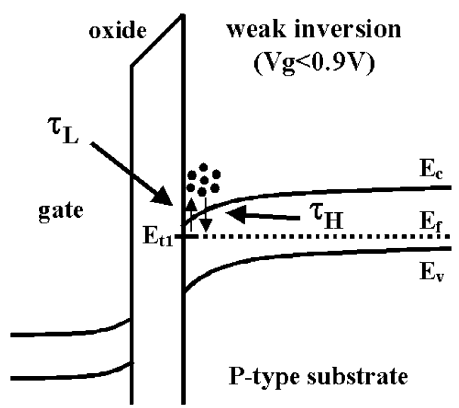

(a)

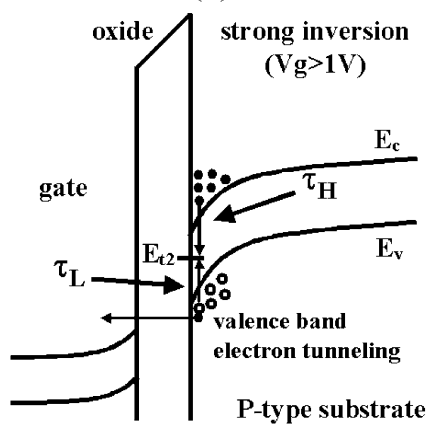

(b)

Fig. 5. (a) Band bending in weak inversion. The RTS results from electron capture $\left(\tau_{H}\right)$ and electron emission $\left(\tau_{L}\right)$ through the interface trap $E_{t 1}$. (b) Band bending in strong inversion. The RTS results from electron capture $\left(\tau_{H}\right)$ and hole capture $\left(\tau_{L}\right)$ at $E_{t 2}$. Channel hole creation due to valence-band electron tunneling is shown.

decreases and $\tau_{L}$ increases because of a larger channel electron population and thus a smaller electron capture time. Our result here is consistent with the findings for thicker gate oxides in previous publications [11]. In contrast, Fig. 6(a) shows the RTS patterns in strong inversion from $V_{g}=1.0$ to $1.6 \mathrm{~V}$. The RTS is still undetectable at $V_{g}=1 \mathrm{~V}$ and reappears for $V_{g}>1 \mathrm{~V}$. Fig. 6(b) shows the $V_{g}$ dependence of average $\tau_{L}$ and $\tau_{H}$. Interestingly, we find that the RTS patterns in strong inversion regime $\left(V_{g}>1 \mathrm{~V}\right)$ exhibit an opposite trend. The $V_{g}$ dependence of $\tau_{H}$ and $\tau_{L}$ in strong inversion is opposite to that in weak inversion. We repeated our measurement on different samples and the opposite trend of the $V_{g}$ dependence is always obtained. Although the mobility fluctuation theory [17] can possibly explain the above opposite gate bias dependence of $\tau_{H}$ and $\tau_{L}$ from weak inversion to strong inversion, further analysis shows that number fluctuation should dominate in the entire range of our measurement gate bias. According to the calculated result in [17, Fig. 3], number fluctuation is dominant in the $V_{g}$ range until $\Delta I_{d} / I_{d}$ changes sign. In our case, the measured $\Delta I_{d} / I_{d}$ versus $V_{g}$ is shown in Fig. 7. The $\Delta I_{d} / I_{d}$ decreases with gate voltage but does not change sign in the entire range of measurement $V_{g}$.

In order to find out the cause of the opposite charge trapping and detrapping behavior from weak inversion to strong inversion, the trap electron occupation factor $\left(f_{t}\right)$ is analyzed. The $f_{t}$ can be evaluated as follows:

$$
f_{t}=\frac{\tau_{L}}{\tau_{L}+\tau_{H}}
$$

Fig. 8(a) shows $f_{t}$ versus $V_{g}$ from weak inversion to strong inversion. In weak inversion regime (i.e., $V_{g}<0.9 \mathrm{~V}$ ), gate oxide

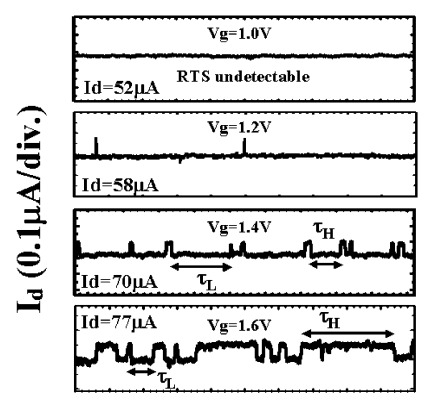

Time (0.1s/div.)

(a)

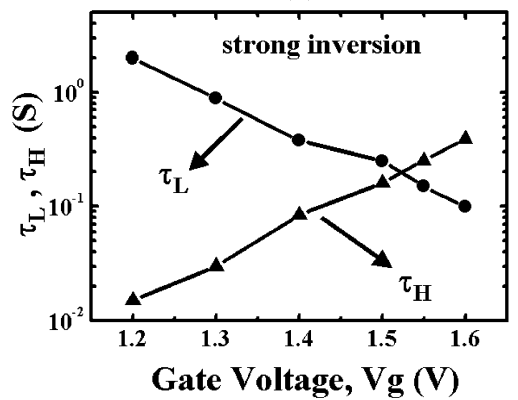

(b)

Fig. 6. (a) Typical RTS patterns at various gate voltages from $V_{g}=1.0$ to $1.6 \mathrm{~V}$ in a small area n-MOSFET $\left(W / L=0.16 / 0.12 \mu \mathrm{m}, t_{\text {ox }}=15 \AA\right)$. (b) Average $\tau_{L}$ and $\tau_{H}$ (extracted from RTS) versus gate voltage in strong inversion regime.

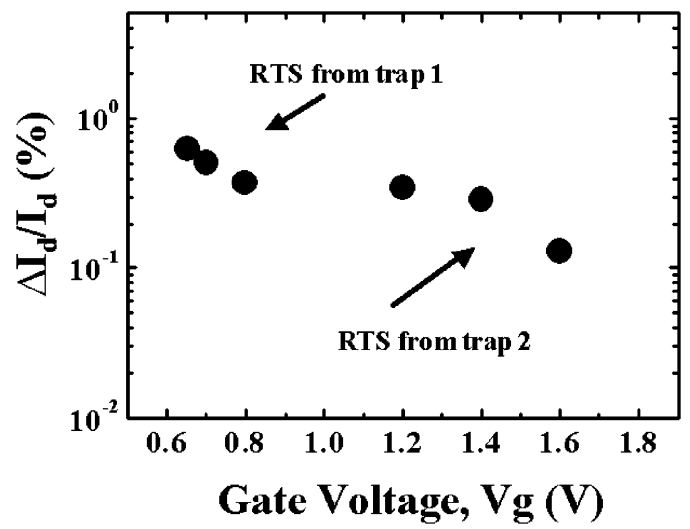

Fig. 7. $\Delta I_{d} / I_{d}$ versus measurement gate bias. The $\Delta I_{d} / I_{d}$ is extracted from the measured RTS.

tunneling is insignificant and the channel is in thermal equilibrium. $f_{t}$ increases with $V_{g}$ because of a larger band-bending. As $f_{t}$ increases to 1 , RTS is undetectable since the trap is always occupied by an electron. However, when $V_{g}$ increases above $1.1 \mathrm{~V}, f_{t}$ begins to decline from unity with increasing $V_{g}$. This means, at a larger $V_{g}$, although the energy level of the interface trap moves more deeply with respect to the electron Fermi level, the chance of the trap being occupied by an electron is smaller. This result is obviously contradicting to the equilibrium case that $f_{t}\left(=1 /\left(1+\exp \left(E_{t}-E_{F}\right) / k T\right)\right)$ should increase as the trap energy becomes more negative with respect to the Fermi level. In other words, the Si channel in strong inversion should be in nonequilibrium condition and the cause is valence-band electron tunneling.

In addition to $f_{t}$, Fig. $8(\mathrm{~b})$ shows the $S_{i d} / I_{d}^{2}$ (measured at $f=100 \mathrm{~Hz}$ in a small area n-MOSFET) from weak inversion to 


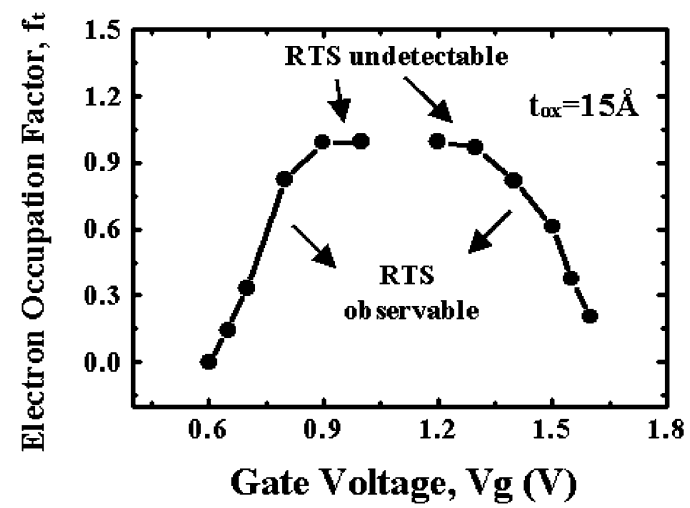

(a)

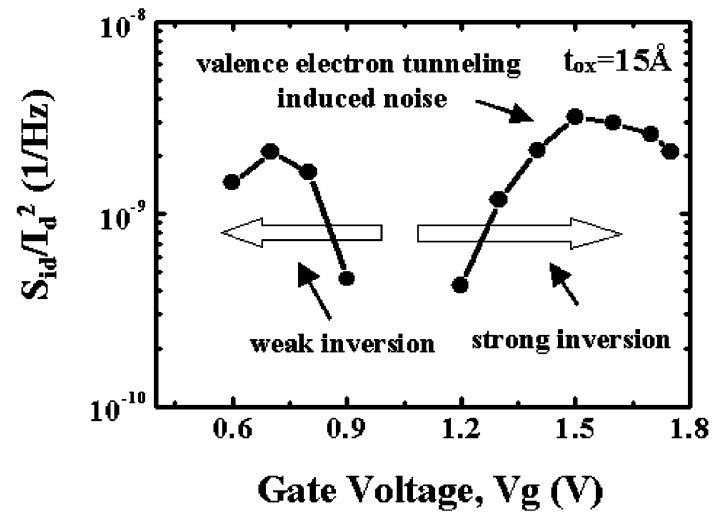

(b)

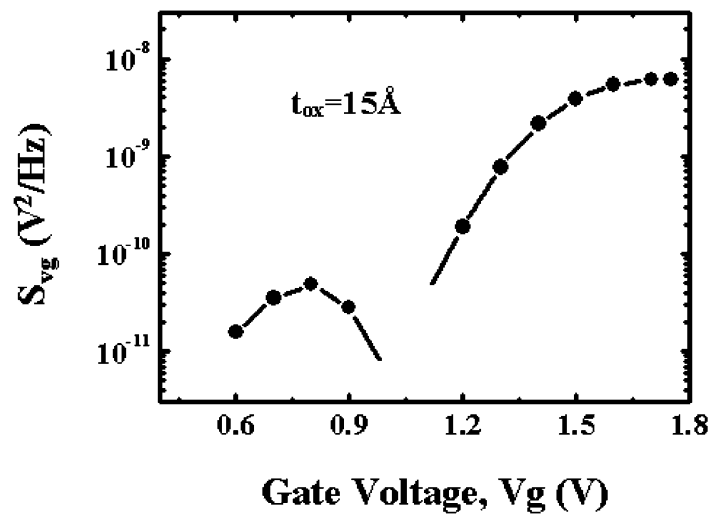

(c)

Fig. 8. (a) Electron occupation factor $\left(f_{t}\right)$, (b) normalized drain current noise, and (c) gate referred voltage noise (measured at $f=100 \mathrm{~Hz}$ ) versus gate voltage in a small area n-MOSFET $\left(W / L=0.16 \mu \mathrm{m} / 0.12 \mu \mathrm{m}, t_{\text {ox }}=15 \AA\right)$.

strong inversion. The readers should be reminded that according to (1) $S_{i d} / I_{d}^{2}$ should have a peak around $f_{t}=0.5$, where $\tau_{L}$ is equal to $\tau_{H}$ and thus $\tau$ reaches a maximum. In Fig. 8(a), $f_{t}$ is 0.5 around $V_{g}=0.75 \mathrm{~V}$ (weak inversion) and $1.5 \mathrm{~V}$ (strong inversion). Thus, $S_{i d} / I_{d}^{2}$ in Fig. 8(b) exhibits two peaks at the above two $V_{g}$. Fig. 8(c) shows the measured $S_{\mathrm{vg}}$ as well. Again, the double hump feature is noticed.

The possible explanation for the abnormal noise behavior in strong inversion is illustrated in Fig. 5(b). In strong inversion regime, a large $V_{g}$ causes strong valence electron tunneling and leaves generated holes behind in the channel. The channel is thus in nonequilibrium. $\tau_{H}$ and $\tau_{L}$ then correspond to electron capture time and hole capture time respectively, as illustrated

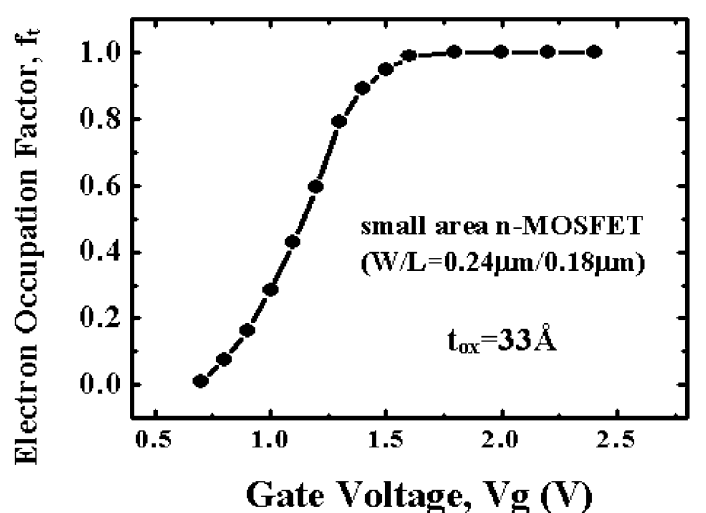

(a)

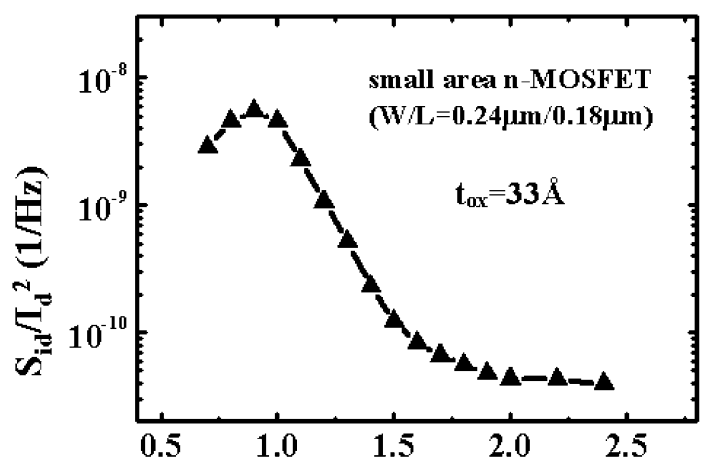

Gate Voltage, Vg (V)

(b)

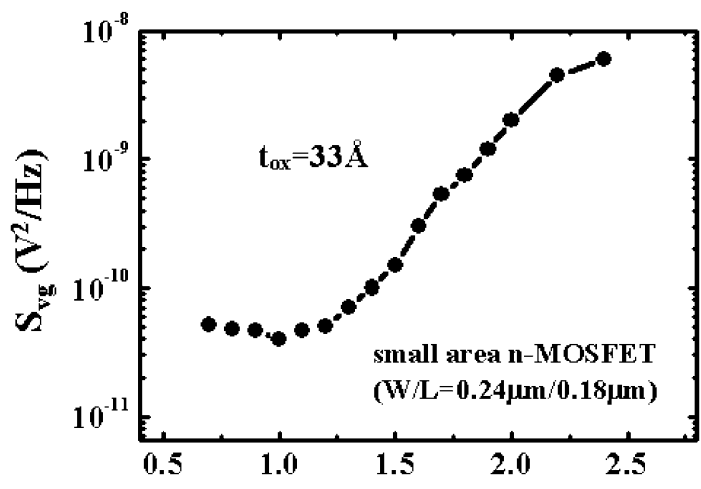

Gate Voltage, Vg (V)

(c)

Fig. 9. (a) Electron occupation factor $\left(f_{t}\right)$, (b) normalized drain current noise, and (c) gate referred voltage noise (measured at $f=100 \mathrm{~Hz}$ ) versus gate voltage in a small area n-MOSFET $\left(W / L=0.24 / 0.18 \mu \mathrm{m}\right.$, and $t_{\text {ox }}=33 \AA$ ).

in Fig. 5(b). Because of the increased channel hole concentration at a larger $V_{g}, \tau_{L}$ is smaller. The nonequilibrium carrier distribution also results in the splitting of electron and hole quasi-Fermi-levels. An interface trap $\left(E_{t 2}\right)$ between the two quasi- Fermi levels can serve as the recombination center of electrons and holes. As a result, the local electron concentration in the vicinity of the trap is reduced and $\tau_{H}$ increases. The increase of $\tau_{H}$ and the decrease of $\tau_{L}$ lead to a reduced $f_{t}$. The second peak of $S_{i d} / I_{d}^{2}$ in strong inversion condition $\left(V_{g}>1 \mathrm{~V}\right)$ in Fig. 8(b) therefore can be well explained. The authors also would like to remark that the Si substrate trap density in bulk 
MOSFETs is around $10^{14} \mathrm{~cm}^{-3}[18]$, or $10^{8} \mathrm{~cm}^{-2}$ by assuming a substrate depletion region width of $10 \mathrm{~nm}$. Although we cannot completely exclude the possibility that the traps stay in the silicon depletion region, the probability that the traps are in the depletion region is very small, as compared to a typical interface trap density of $10^{10} \mathrm{~cm}^{-2}$.

For comparison, the $f_{t}$ and the noise power density versus $V_{g}$ in a thicker gate oxide ( $33 \AA$ ) n-MOSFET are also characterized. The result is shown in Fig. 9. The $f_{t}$ stays at unity in strong inversion. Neither RTS nor the double hump feature in noise power density is observed in strong inversion since valence-bane tunneling is insignificant in such thick gate oxide devices.

\section{CONCLUSION}

We identified two low-frequency noise sources in ultrathin oxide (15- $\AA$ ) n-MOSFETs. In weak inversion, the noise arose from electron capture and emission at a shallower interface trap. In strong inversion, we observed an abnormal increase in low-frequency noise. This abnormal noise behavior is not observed in a thicker gate oxide ( $33 \AA$ ) device, where valence-band tunneling is insignificant. The traditional flicker noise model based on oxide charge tunnel trapping/detrapping cannot account for this excess low-frequency noise. The analysis of RTS patterns and trap occupation factor reveals that the channel is in nonequilibrium at a large gate voltage due to valence-band tunneling. The increased channel hole concentration and a Fermi-level splitting caused by valence-band electron tunneling should be responsible for the excess low-frequency noise.

\section{ACKNOWLEDGMENT}

The authors would like to thank TSMC, Taiwan, for providing technical support.

\section{REFERENCES}

[1] A. van der Ziel, Noise in Solid State Devices and Circuits. New York: Wiley, 1986.

[2] A. Hajimiri and T. H. Lee, "A general theory of phase noise in electrical oscillators," IEEE J. Solid-State Circuits, vol. 33, no. 2, pp. 179-194, Mar. 1998.

[3] K. K. Hung, P. K. Ko, C. Hu, and Y. C. Cheng, "A unified model for the flicker noise in metal-oxide-semiconductor field-effect transistors," IEEE Trans. Electron Devices, vol. 37, no. 5, pp. 654-665, May 1990.

[4] —- "A physics-based MOSFET noise model for circuit simulators," IEEE Trans. Electron Devices, vol. 37, no. 10, pp. 1323-1333, Oct. 1990.

[5] M. H. Tsai and T. P. Ma, " $1 / f$ noise in hot-carrier damaged MOSFETs: Effects of oxide charge and interface traps," IEEE Electron Device Lett., vol. 14, no. 4, pp. 256-258, Apr. 1993.

[6] M. J. Knitel, P. H. Woerlee, A. J. Scholten, and A. T. A. Zegers-Van Duijnhoven, "Impact of process scaling on $1 / f$ noise in advanced CMOS technologies," in IEDM Tech. Dig., 2000, pp. 463-466.

[7] H. S. Momose, H. Kimijima, S.-I. Ishizuka, Y. Miyahara, T. Ohguro, T. Yoshitomi, E. Morifuji, S.-I. Nakamura, T. Morimoto, Y. Katsumata, and H. Iwai, "A study of flicker noise in n- and p-MOSFETs with ultrathin gate oxide in the direct-tunneling regime," in IEDM Tech. Dig., 1998, pp. 923-926.

[8] A. Mercha, J. M. Rafí, E. Simoen, E. Augendre, and C. Claeys, “'Linear kink effect' induced by electron valence-band tunneling in ultrathin gate oxide bulk and SOI MOSFETs," IEEE Trans. Electron Devices, vol. 50, no. 11, pp. 1675-1682, Nov. 2003.

[9] J. W. Wu, J. W. You, H. C. Ma, C. C. Cheng, C. S. Chang, G. W. Huang, and T. Wang, "Valence-band tunneling induced low-frequency noise in ultrathin oxide (15- $\AA$ ) n-type metal-oxide-semiconductor field effect transistors," Appl. Phys. Lett., vol. 85, pp. 5076-5077, 2004.
[10] J. W. Wu, H. C. Ma, C. C. Cheng, G. W. Huang, C. S. Chang, and T. Wang, "Low frequency noise degradation in ultrathin oxide $(15-\AA)$ analog nMOSFETs resulting from valence-band tunneling," in Proc. Int. Reliab. Phys. Symp., 2005, pp. 260-264.

[11] K. Kandiah, M. O. Deighton, and F. B. Whiting, "A physical model for random telegraph signal currents in semiconductor devices," J. Appl. Phys., vol. 66, pp. 937-948, 1989.

[12] N. V. Amarasinghe and Z. Çelik-Butler, "Complex random telegraph signals in $0.06 \mu \mathrm{m}^{2}$ MDD n-MOSFETs," Solid State Electron., vol. 44, pp. 1013-1019, 2000.

[13] Z. Çelik-Butler and F. Wang, "Effects of quantization on random telegraph signals observed in deep-submicron MOSFETs," Microelectron. Reliab., vol. 40, pp. 1823-1831, 2000.

[14] G. Ghibaudo and T. Boutchacha, "Electrical noise and RTS fluctuations in advanced CMOS devices," Microelectron. Reliab., vol. 42, pp. 573-582, 2002.

[15] E. Simoen and C. Claeys, "Random telegraph signal: A local probe for single point defect studies in solid-state devices," Mater. Sci. Eng., vol. B91-92, pp. 136-143, 2002.

[16] C. W. Tsai, S. H. Gu, L. P. Chiang, and T. Wang, "Valence-band tunneling enhanced hot carrier degradation in ultrathin oxide nMOSFETs," in IEDM Tech. Dig., 2000, pp. 139-142.

[17] S. T. Martin, G. P. Li, E. Worley, and J. White, "The gate bias and geometry dependence of random telegraph signal amplitudes," IEEE Trans. Electron Devices, vol. 18, no. 3, pp. 444-446, Mar. 1997.

[18] D. S. Ang, Z. Lun, and C. H. Ling, "Generation-recombination noise in the near fully depleted SIMOX SOI nMOSFETs-Physical characteristics and modeling," IEEE Trans. Electron Devices, vol. 50, no. 12, pp. 2490-2498, Dec. 2003.

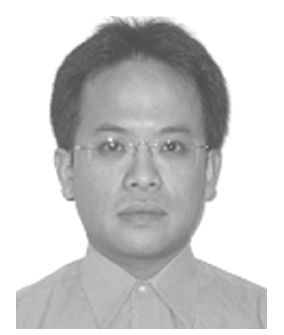

Jun-Wei Wu (S'02) was born in Tao-Yuan, Taiwan, R.O.C. He received the B.S. and Ph.D. degrees in electronics engineering from National Chiao-Tung University, Hsinchu, Taiwan, in 1998 and 2004, respectively.

From 2004 to 2005, he was with Macronix International Company, Ltd. (MXIC), Hsinchu, where he worked on MOS device modeling. Since 2005, he has been with Taiwan Semiconductor Manufacturing Company (TSMC), Ltd., Hsinchu, where he has worked on RF CMOS device optimization and characterization, including RF noise, flicker noise, and device mismatch.

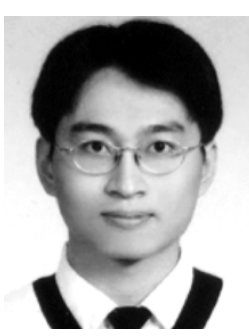

Jian-Wen You was born in Kao-Hsiung, Taiwan, R.O.C., in 1979. He received the B.S. degree in engineering science from National Cheng-Kung University Tainan, Taiwan, in 2002 and the M.S. degree in electronics engineering from National Chiao-Tung University, Hsinchu, Taiwan, in 2004.

In 2004, he joined United Microelectronics Corporation (UMC), Hsinchu. His research interest includes the reliability issues and analog performance of CMOS.

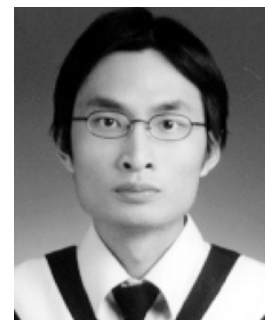

Huan-Chi Ma was born in Tainan, Taiwan, R.O.C., in 1981. He received the B.S. and M.S. degrees in electronics engineering from National Chiao-Tung University, Hsinchu, Taiwan, in 2003 and 2005, respectively, where he is currently pursuing the $\mathrm{Ph} . \mathrm{D}$. degree.

His research interest includes flicker noise characterization and modeling. 


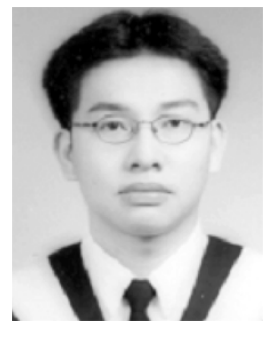

Chih-Chang Cheng received the B.S. degree in physics from National Central University, Taoyuan, Taiwan, R.O.C., in 2002. He is currently pursuing the Ph.D. degree in electronics engineering at the National Chiao-Tung University, Hsinchu, Taiwan.

His research interests include high-voltage power devices and flicker noise.

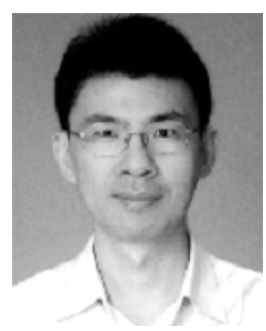

Chang-Feng Hsu received the B.S. degree from National Ocean University, Kee-Lung, Taiwan, R.O.C., in 1986, and the M.S. degree in electronics engineering from National Chiao-Tung University (NCTU), Hsinchu, Taiwan, in 1990, where he is currently pursuing the $\mathrm{Ph}$.D. degree.

From 1990 to 1998, he was with the Department of Electrical Engineering, Ming-Hsin University of Technology, Hsinchu, as an Instructor. From 1998 to 2002, he was with the Department of VLSI Technology Development, Etron Technologies Inc., Hsinchu, as a Member of Technical Staff where he was engaged in the development of memory devices design and modeling. From 2002 to 2004, he was with the Department of Technology Development, SiS Technologies Inc., Hsinchu, as a Technical Manager, where he was engaged in the development of deep submicrometer devices and RF devices and circuits. Since 2004, he has been with Taiwan Microelectronics Technologies Inc., Hsinchu. His research interests include reliability issues and hot carrier effects in deep submicrometer MOSFETs and RF CMOS device modeling, flicker noise characterization, and modeling.

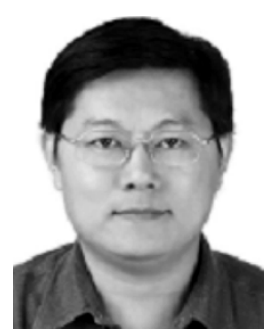

Chih-Sheng Chang was born in Hualien, Taiwan, R.O.C., on November 3, 1964. He received the B.S. and M.S. degrees in electrical engineering from National Taiwan University, Taipei, Taiwan, in 1986 and 1990, respectively, and the Ph.D. degree from the Department of Electrical and Computer Engineering, University of Illinois, Urbana-Champaign, in 1996.

Since 1999, he has been with Taiwan Semiconductor Manufacturing Company (TSMC), Ltd., Hsinchu, where he has worked on TCAD, MOS device design, and RF devices optimization and characterization. He has been the main device designer for TSMCs $0.15-\mu \mathrm{m}$ and $0.13-\mu \mathrm{m}$ high-speed devices. Currently, he is a Technology Manager in the Communication Technology Department, Logic Technology Division, where he is in charge of the development of RF active and passive devices for 0.18-, $0.13-$, and $90-\mathrm{nm}$ technologies.

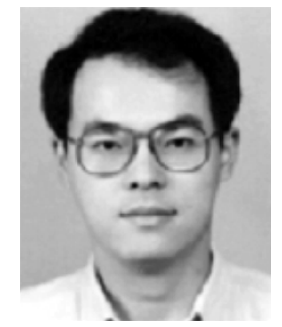

Gou-Wei Huang was born in Taipei, Taiwan, R.O.C., in 1969. He received the B.S. degree in electronics engineering and the Ph.D. degree from National Chiao-Tung University, Hsinchu, Taiwan, in 1991 and 1997, respectively.

He joined National Nano Device Laboratories, Hsinchu, in 1997 as an Associate Researcher. His current research interests focus on microwave device design, characterization, and modeling.

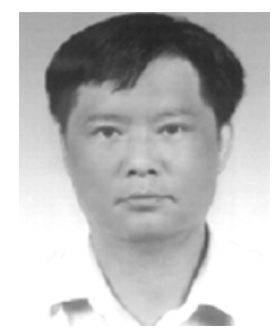

Tahui Wang (S'85-M'86-SM'94) was born in TaoYuan, Taiwan, R.O.C., on May 3, 1958. He received the B.S.E.E. degree from National Taiwan University, Taipei in 1980, and the Ph.D. degree in electrical engineering from the University of Illinois, Urbana-Champaign, in 1985.

From 1985 to 1987, he was with Hewlett-Packard Laboratories, Palo Alto, CA, where he was engaged in the development of GaAs HEMT devices and circuits. Since 1987, he has been with the Department of Electronics Engineering, National Chiao-Tung University, Hsinchu, Taiwan, where he is currently a Professor. His research interests include hot carrier phenomena characterization and reliability physics in VLSI devices, RF CMOS devices, and nonvolatile semiconductor devices.

Dr. Wang was granted the Best Teacher Award by the Ministry of Education, Taiwan, R.O.C. He has served as technical committee member of many international conferences, including IEDM, IRPS, and VLSI-TSA. His name was listed in Who's Who in the World. 